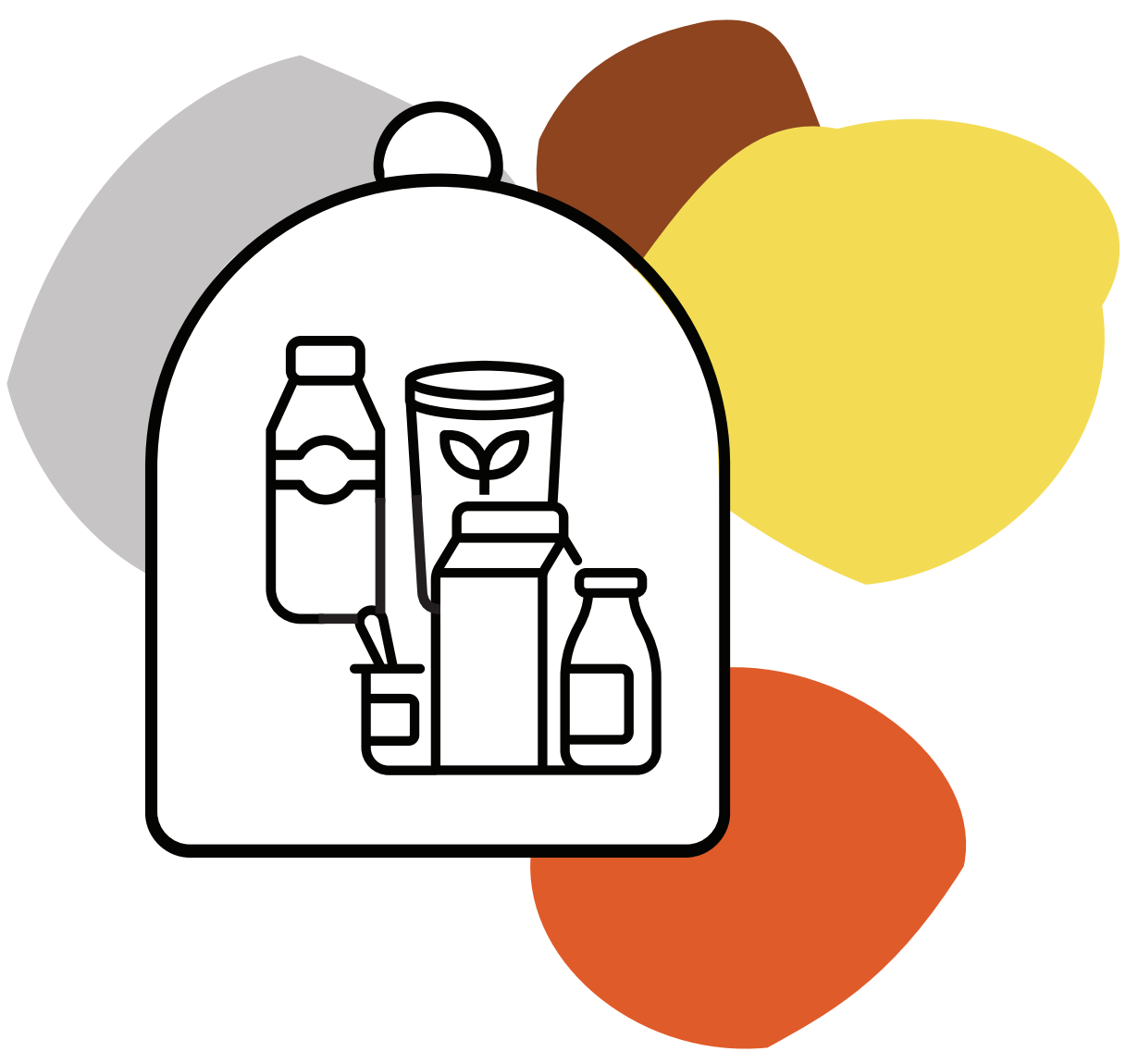

\title{
APORTES DEL ENFOQUE SISTEMÁTICO PARA EL ASEGURAMIENTO DE LA INOCUIDAD ALIMENTARIA
}

\author{
AUTORES \\ CINTYA GONZÁLEZ (1) \\ DANIELA ESCOBAR (2) \\ VERÓNICA SKERL (3) \\ MERCEDES ALBISTUR (3) \\ DANIEL PIPPOLO (3) \\ nivel mundial, los consumidores valoran \\ cada vez con más fuerza aspectos relacio- \\ nados con la inocuidad alimentaria. Esto de- \\ termina la necesidad de elaborar productos \\ seguros y de buena calidad, lo que implica una adecuada \\ gestión de compra de las materias primas e insumos, el \\ aseguramiento de la eficiencia y el desempeño de los \\ procesos, el cumplimiento de las especificaciones y el \\ mantenimiento de las evidencias de los controles rea- \\ lizados a lo largo de todo el proceso. Asimismo, en el \\ contexto actual existe una creciente competencia; los
}

(1) LÁCTEOS DOÑA ÁNGELA, ASUNCIÓN, PARAGUAY

(2) LATITUD FUNDACIÓN LATU, MONTEVIDEO, URUGUAY

(3) GERENCIA DE TECNOLOGÍA Y GESTIÓN, LATU, MONTEVIDEO, URUGUAY 
productos y mercados cambian rápidamente y se torna cada vez más importante para la empresa establecer herramientas de control de gestión modernas y asumir la gestión del conocimiento como bien competitivo. Para alcanzar el éxito sostenido de la organización es necesario monitorear factores críticos y motivar la creatividad e iniciativa de los empleados.

En este trabajo se analiza la relevancia de diseñar e implementar un sistema de gestión de la inocuidad alimentaria en una industria láctea y se evalúan globalmente los beneficios obtenidos para atender los desafíos internos y externos. El análisis sistemático de todos los procesos de una empresa y su relación con los requisitos de las normas de gestión impacta positivamente en las capacidades de la organización para gestionar en forma efectiva todos sus procesos. A su vez, esta práctica permite asegurar la calidad e inocuidad de los productos entregados a los clientes, así como la optimización de los recursos, lo que incide finalmente en la rentabilidad de la empresa.

\section{En contexto}

Lácteos Doña Ángela surgió en 1955 como unidad de producción lechera que tenía como actividad principal el ordeñe de leche y su posterior distribución y comercialización en Asunción, bajo la modalidad de "venta de leche casa por casa". Con el transcurso del tiempo, la producción comenzó a aumentar y se desarrollaron nuevos canales de comercialización, lo que junto con el permanente afán de superación de la empresa determinaron la necesidad de industrializar la producción propia. El $1^{\circ}$ de julio de 2011 comenzó a operar la nueva planta industrial que se encuentra instalada en un predio de 30 hectáreas y con una capacidad de producción de 125.000 litros por día.

Durante el período de abril de 2016 a julio de 2017, Lácteos Doña Ángela diseñó e implementó los requisitos de un sistema de gestión de la inocuidad alimentaria según el esquema de certificación FSSC, el cual combina los requisitos de Norma de Gestión de Seguridad Alimentaria ISO 22000 (International Organization for Standarization, 2005), requisitos para cualquier organización de la cadena alimentaria, con las especificaciones técnicas de la ISO/TS 22002-1 (International Organization for Standarization, 2009) (programas de pre-requisitos) y los requisitos adicionales de Global Food Safety Initiative (GSF; ver enlaces recomendados). En julio de 2017 recibió la auditoría externa por parte de un organismo certificador acreditado, que verificó in situ el cumplimiento de todos los requisitos y recomendó la certificación bajo el esquema de FSSC 22000.

El objetivo de este artículo es describir y examinar la metodología de una investigación sistémica utilizada para el análisis de los requisitos normativos, el diseño y posterior implementación de los requisitos de un sistema de gestión de la inocuidad. Se identifican algunos hitos y posteriormente se presentan los beneficios obtenidos y una serie de recomendaciones derivadas de las lecciones aprendidas. En particular, se hace énfasis en la importancia de la evaluación de los resultados analíticos de la calidad microbiológica de la leche en las distintas etapas del proceso productivo. Esto permite explorar las causas y proponer algunas mejoras aplicadas a lo largo de toda la cadena de producción con el objetivo de obtener productos lácteos de calidad e inocuidad, desde la recepción de materia prima, a la elaboración y también durante toda su vida útil.

\section{Metodología y desarrollo}

Con base en el concepto de comunidades de práctica (grupo donde se generan y toman decisiones, se aprende y se difunde el conocimiento; Pippolo y Di Candia, 2009) el equipo de inocuidad alimentaria de la empresa, acompañado por los consultores del LATU, conformó un grupo de trabajo para evaluar el entorno interno y externo y definir objetivos, acciones, responsables y plazos para el cumplimiento de las acciones. El equipo estuvo integrado por responsables de diferentes áreas de la organización con el fin de comenzar a generar una única visión de la empresa, eliminar viejos prejuicios entre diferentes clientes y proveedores internos y asegurar la alineación en los objetivos y lineamientos principales.

Trascurridos los primeros seis meses de trabajo, y una vez que la empresa contaba con una base documental y operativa de las buenas prácticas de manufactura (BPM) en todo el proceso y con planes de análisis de peligros y puntos críticos de control (APPCC) para los diferentes productos, el equipo profundizó un estudio sistemático de la calidad de la leche a lo largo del proceso productivo y su efecto en la calidad final del producto, utilizando el análisis de causa efecto (Figura 1). 


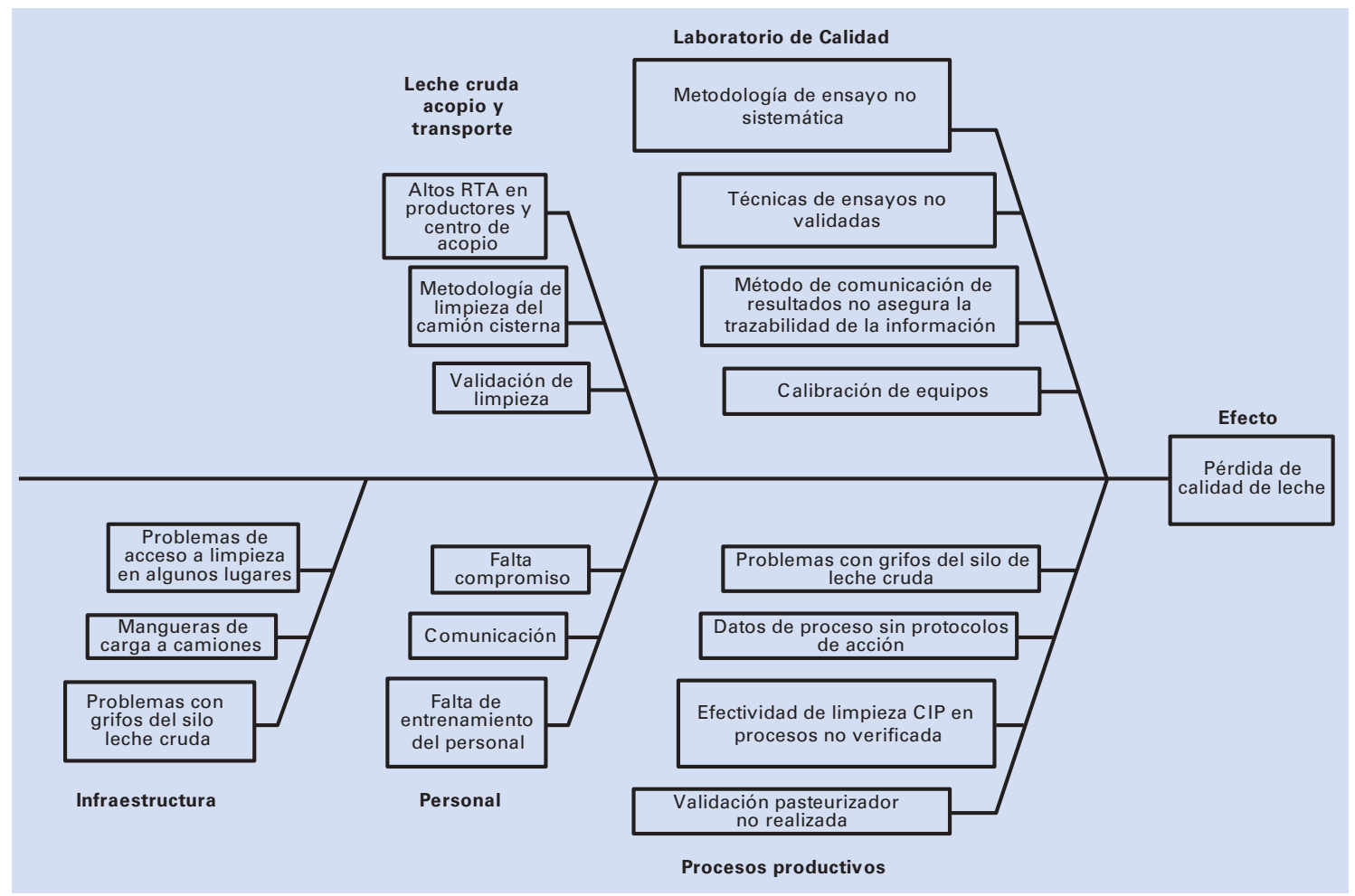

Figura 1. Análisis de causas en pérdida de calidad de leche.

A partir de este análisis se identificó la necesidad de realizar un estudio de los resultados microbiológicos históricos que presentaba la leche en las distintas etapas del proceso (desde su acopio, transporte y proceso de producción), así como para los productos elaborados. A su vez, se examinaron los procedimientos del laboratorio y del personal, los problemas que pudiesen provenir de la infraestructura de la planta, y se fueron aplicando los criterios de exigencia de la norma para el aseguramiento de la inocuidad alimentaria.

Del análisis de resultados microbiológicos históricos se desprendió la necesidad de diseñar un plan de muestreo para profundizar en el análisis de condiciones de recepción y resultados de microorganismos. Con este insumo se planificó el establecimiento de acciones, verificaciones y validaciones.

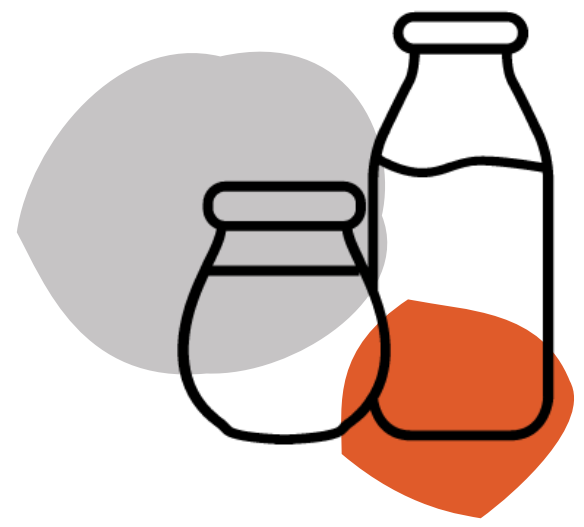




\begin{tabular}{|c|c|c|c|c|c|c|c|c|}
\hline \multirow{2}{*}{ Análisis: } & \multirow{2}{*}{ Método } & \multirow{2}{*}{$\begin{array}{l}\text { Temp. } \\
{ }^{\circ} \mathrm{C}\end{array}$} & \multirow{2}{*}{$\begin{array}{c}\text { Tiempo } \\
\text { (hs) }\end{array}$} & \multicolumn{2}{|c|}{ Leche } & \multirow{2}{*}{$\begin{array}{c}\text { Producto } \\
\text { final }\end{array}$} & \multirow{2}{*}{$\begin{array}{l}\text { Agua de } \\
\text { lavados }\end{array}$} & \multirow{2}{*}{$\begin{array}{l}\text { Hisopado } \\
\text { en } \\
\text { camiones }\end{array}$} \\
\hline & & & & Cruda & Procesada & & & \\
\hline $\begin{array}{c}\text { Recuento } \\
\text { Aeróbios } \\
\text { Mesófilos (RTA) }\end{array}$ & $\begin{array}{l}\text { Petrifilm AOAC } \\
\text { método oficial } \\
986.33\end{array}$ & $(32 \pm 1)$ & $(48 \pm 3)$ & $x$ & $x$ & $x$ & $x$ & $x$ \\
\hline $\begin{array}{c}\text { Recuento } \\
\text { Coliformes (CT) }\end{array}$ & $\begin{array}{c}\text { Petrifilm AOAC } \\
\text { método oficial } \\
986.33 \text { y } 989.10\end{array}$ & $(32 \pm 1)$ & $(24 \pm 2)$ & & $X$ & $x$ & $x$ & $x$ \\
\hline Bioluminiscencia & 3MClean Trace & & & & & & & $x$ \\
\hline \multicolumn{4}{|c|}{ Momento/Punto de muestreo } & $\begin{array}{c}\text { Al comple- } \\
\text { tar la carga } \\
\text { Al inicio de } \\
\text { la pasteuri- } \\
\text { zación }\end{array}$ & & $\begin{array}{l}\text { Al final } \\
\text { de la vida } \\
\text { útil }\end{array}$ & & $\begin{array}{l}\text { Tanque y } \\
\text { acoplado }\end{array}$ \\
\hline
\end{tabular}

Tabla 1. Plan de muestreo y métodos analíticos utilizados.

En la Tabla 1 se presenta el plan de muestreo realizado y los métodos analíticos utilizados.

En virtud del análisis de causas y de los resultados analíticos, el equipo de inocuidad decidió intervenir en las siguientes etapas del proceso:

1) Previo a la entrada de la planta industrial: se tomaron medidas que involucran a los establecimientos de producción de leche, su transporte y recepción.

- Productores de leche: El asesoramiento a tambos se focalizó en recomendar mejoras a realizar en los establecimientos con el fin de obtener leche cruda de buena calidad para procesar. Esto se complementó con un plan anual de seguimiento a los establecimientos lecheros para verificar la ejecución de las acciones recomendadas y evaluar los resultados obtenidos

- Silos de recibo de leche cruda en la planta industrial: Se mejoró la conexión del grifo del silo, donde se retira la muestra de leche para ser analizada. Se modificaron las conexiones de colector de descarga de cisternas de transporte de leche y pulido de conexiones de mangueras de descarga.

- Camión cisterna: Se reubicó la bomba de retorno de lavado CIP de camiones de leche, se repararon las tapas de entrada de hombre y el pulido de unión de soldadura de respiraderos de cisternas de transporte de leche.
2) En la planta industrial:

- Proceso de limpieza y sanitización, especialmente en el clean in place (cambio de mangueras). Se modificaron los procedimientos de limpieza y se actualizó el procedimiento de comunicaciones y registros.

- Higiene y sanitización a través de los procedimientos operativos estandarizados de saneamiento (establecimiento y comunicación de límites de control en monitoreo mediante hisopado de superficie en contacto directo e indirecto con el alimento). 


\section{Resultados analíticos}

En la Tabla 2 se presentan los resultados analíticos según el plan de muestreo. Para los resultados de recuento total de aerobios (RTA) se toman como referencia los valores máximos de las normas paraguayas (Instituto Nacional de Tecnología, Normalización y Metrología, 2008 y 2010).

\begin{tabular}{|c|c|c|c|c|}
\hline Muestra & análisis & antes & después & \% disminución \\
\hline Leche cruda al arribo a planta & Media RTA 1 & $20,0 \%$ & $7,9 \%$ & $60,7 \%$ \\
\hline \multirow{2}{*}{ Muestras de hisopado camión } & Fuera rango $\mathrm{CT}$ & 3 & 0 & $-/ /-$ \\
\hline & Fuera rango $\mathrm{RTA}$ & 8 & 0 & $-/ /-$ \\
\hline \multirow{2}{*}{ Muestras de agua lavado camión } & Fuera rango $\mathrm{CT}$ & 3 & 0 & $-/ /-$ \\
\hline & Fuera rango RTA & 14 & 0 & $-/ /-$ \\
\hline \multirow{2}{*}{ Leche envasada } & Fuera rango $\mathrm{CT}$ & 0 & 0 & $-/ /-$ \\
\hline & Media RTA ${ }^{1}$ & $56,2 \%$ & $34,2 \%$ & $39,1 \%$ \\
\hline \multirow{2}{*}{ Leche al fin de vida útil } & Fuera rango $\mathrm{CT}$ & 0 & 0 & $-/ /-$ \\
\hline & Media RTA1 & $42,3 \%$ & $36,2 \%$ & $14,5 \%$ \\
\hline
\end{tabular}

Tabla 2. Resultados analíticos según el plan de muestreo.

Los resultados analíticos evidencian una importante disminución de los valores en los siguientes productos:

- Leche cruda: Si bien la empresa partía de un valor de RTA muy bajo para los requisitos normativos de Paraguay (20\% del valor máximo), a partir de las mejoras propuestas por el equipo de inocuidad se alcanzó una mejora sustancial en la calidad de la leche cruda recibida, con una reducción del 60 \% en los recuentos de aerobios totales (RTA) y se eliminó la presencia de valores fuera de rango para coliformes totales (CT) para todas las muestras analizadas (Gráfico 1).

- Camiones cisterna: Las acciones realizadas determinaron la eliminación de la presencia de valores fuera de rango para hisopado de camión tanto en CT y RTA, así como para el agua de lavado (Gráficos 2 y 3).
- Leche pasteurizada envasada y al final de su vida útil: La empresa parte siempre de valores inferiores a los establecidos por la norma paraguaya (Instituto Nacional de Tecnología, Normalización y Metrología, 2010), en el entorno del $50 \%$ y $40 \%$, respectivamente. Igualmente, se constató una mejora en los valores obtenidos en base al análisis y acciones propuestas por el equipo de inocuidad, que descendieron $40 \%$ y $15 \%$ con respecto a los valores previos a la intervención del equipo (Gráficos 4 y 5). 


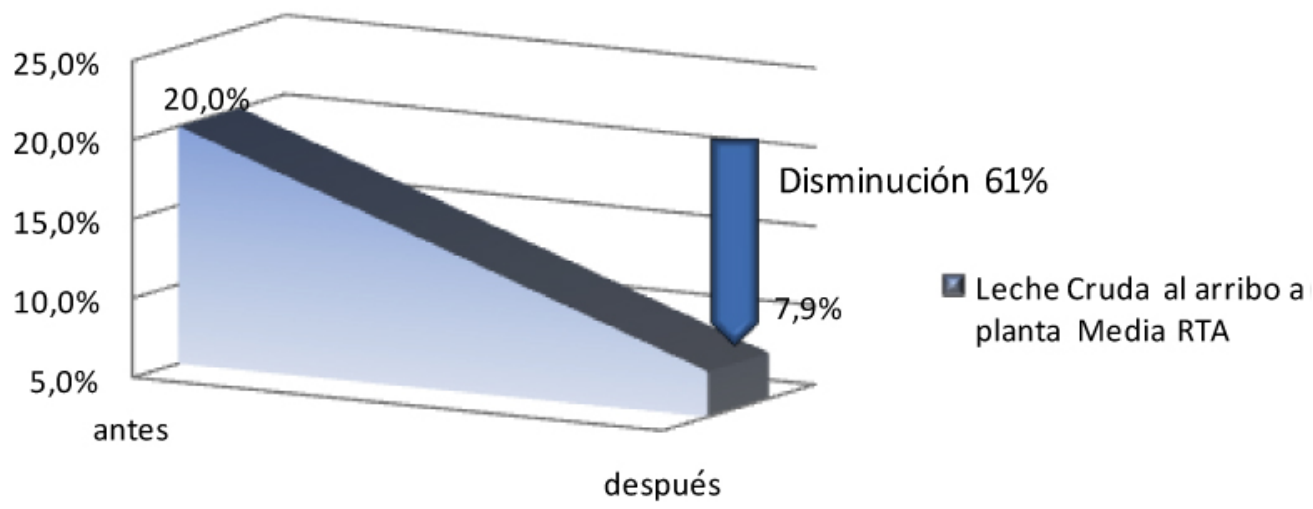

Gráfico 1. Recuentos de aerobios totales (RTA) de leche cruda.

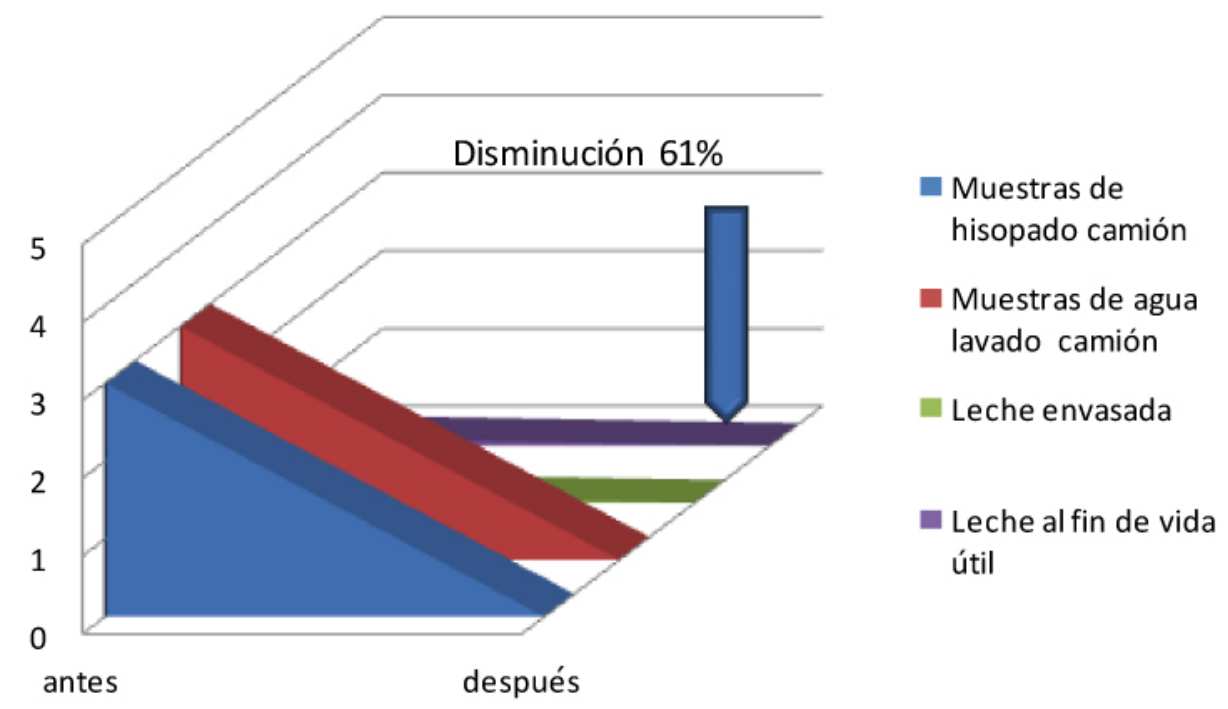

Gráfico 2. Coliformes totales (CT) fuera de rango.

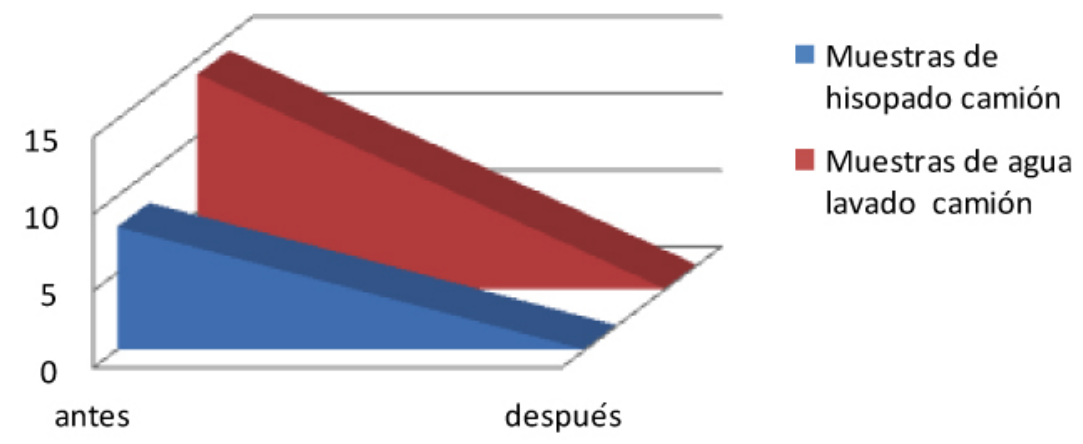

Gráfico 3. Recuentos de aerobios totales (RTA) fuera de rango. 


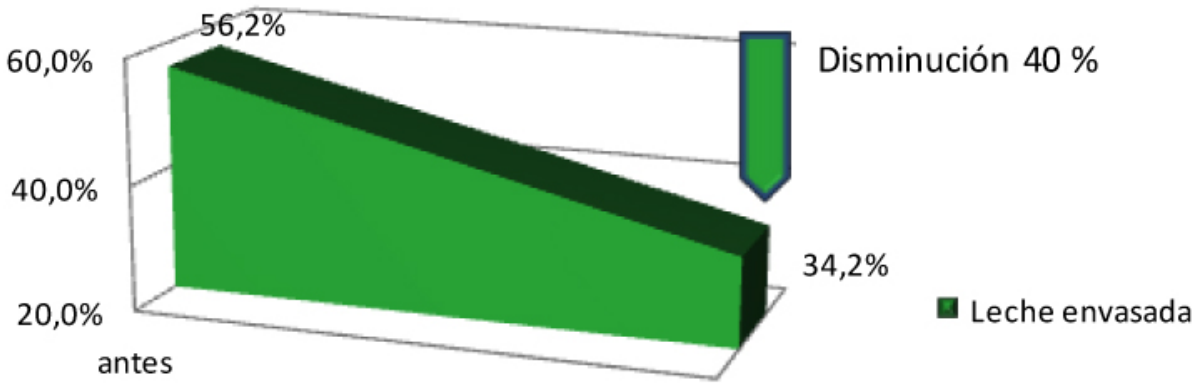

después

Gráfico 4. Recuentos de aerobios totales (RTA) de leche envasada.

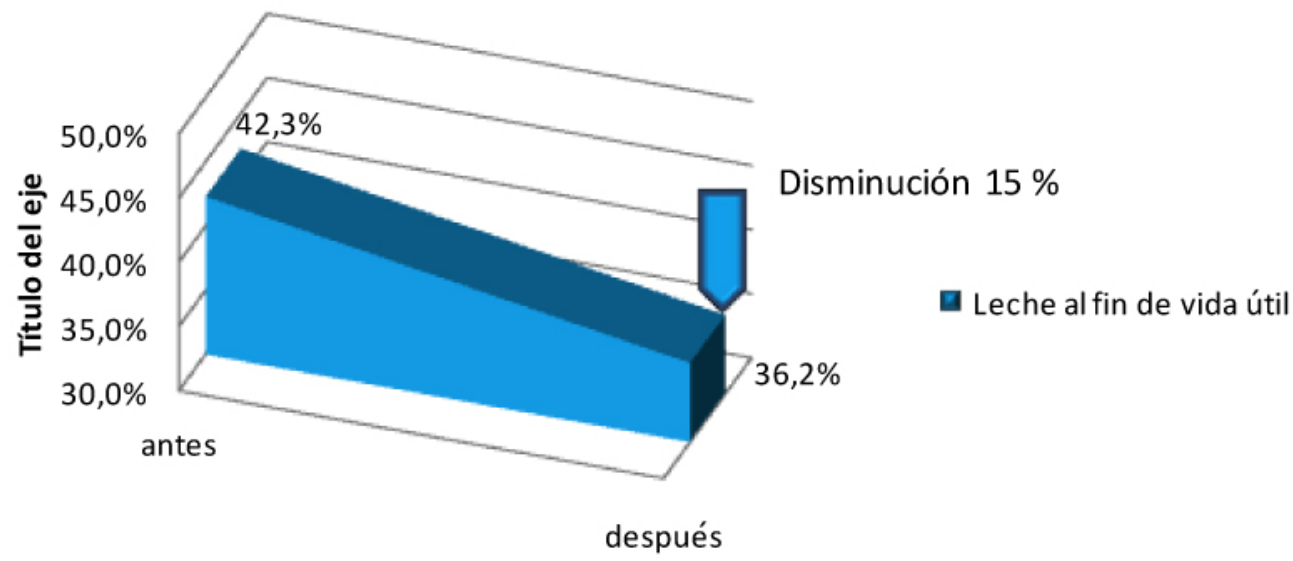

Gráfico 5. Recuentos de aerobios totales (RTA) de la leche fin de vida útil.

\section{Primeras conclusiones}

Los resultados presentados muestran la importancia de contar con un equipo que centralice la implementación de un sistema de gestión de inocuidad alimentaria (SGIA) y su uso como herramienta para la mejora de los procesos. Un análisis sistémico como el realizado por la comunidad de práctica determinó la planificación de acciones que involucraron a todos los actores y aspectos de los procesos productivos, desde los productores de leche a la mejora de infraestructura, y que promovieron cambios en los procedimientos de higiene y monitoreo de limpieza y desinfección, entre otros. Si bien en este trabajo se presentan los resultados para el producto leche envasada, se observaron las mismas tendencias para todos los productos elaborados por la empresa.

El diseño e implementación de un SGIA en la organización fue útil para analizar y establecer acciones sobre los efectos de la calidad de la materia prima y de las etapas del proceso productivo. Además, permitió obtener mejoras objetivas en la calidad e inocuidad de los productos ofrecidos a los clientes de la empresa.

La implementación del SGIA conllevó sistematizar y poner a punto el plan de muestreo y los ensayos de control en planta, lo que habilitó dos beneficios. Primero, reducir el riesgo a incidencias; mejoró la calidad de la materia prima recibida en planta y la calidad de los procesos (higiene y mantenimiento), lo cual se confirma en los resultados obtenidos tanto para RTA como CT. Segundo, brindar una mayor confianza a los clientes en cuanto a la inocuidad de los alimentos. La implementación de las mejores prácticas de la industria de fabricación de alimentos derivó en una mayor seguridad alimentaria. 


\section{Reconocimientos}

El equipo desea agradecer a Marianela Cremona por sus aportes en el estudio de causas, planificación de los muestreos y análisis de datos posteriores, y a Natalia Baez y Luz Pereira por el desarrollo de los planes de muestreos y análisis complementarios.

\section{Referencias}

Instituto Nacional de Tecnología, Normalización y Metrología, 2008. NP 25011 83: Leche cruda. Requisitos generales. Asunción: INTN.

Instituto Nacional de Tecnología, Normalización y Metrología, 2010. NP. 25026 84: Leche pasteurizada. Requisitos Generales. Asunción: INTN.

International Organization for Standarization, 2005. ISO 22000: Food safety management systems - Requirements for any organization in the food chain. Ginebra: ISO.

International Organization for Standarization, 2009. ISO/ TS 22002-1: Prerequisite programmes on food safety Part 1: Food manufacturing. Ginebra: ISO.

Pippolo, Daniel y di Candia, Carina, 2009. Impacto de las herramientas de gestión en la conducción de las empresas. En: Innotec, 1, pp.26-33

\section{Referencias recomendadas}

Comisión del Codex Alimentarius, 1999. Código internacional recomendado de prácticas principios generales de higiene de los alimentos, CAC/RCP-1 (1969), Rev. 3 (1997), enmendado en 1999. Roma: Comisión del Codex Alimentarius

\section{Enlaces recomendados}

Global Food Safety Iniciative: https://www.mygfsi.com 